\title{
An Agent-based Simulation for Studying Air Pollution from Traffic in Urban Areas: The Case of Hanoi City
}

\author{
KAFANDO Rodrique ${ }^{1}$, HO Tuong Vinh ${ }^{2}$, NGUYEN Manh Hung ${ }^{3}$ \\ ${ }^{1,2}$ IFI, Vietnam National University in Hanoi, Vietnam \\ ${ }^{2,3}$ UMI UMMISCO 209 (IRD/UPMC), Hanoi, Vietnam \\ ${ }^{3}$ Posts and Telecommunications Institute of Technology (PTIT), Vietnam
}

\begin{abstract}
In urban areas, traffic is one of the main causes of air pollution. Establishing an effective solution to raise public awareness of this phenomenon could help to significantly reduce the level of pollution in urban areas. In this study, we design and implement an agent-based simulation allowing to study the principles of production and dispersion of pollutants from road traffic in urban areas. The simulation takes into account different factors that can produce pollutants from the urban zone (the case of Hanoi city in Vietnam): roads and streets, vehicles (types, quantity), traffic, wind direction, etc. With this simulation, one can observe and study the emission and dispersion of pollutants from traffic by conducting experiments with various scenarios and parameters. This work is an interesting solution to sensitize the public's awareness on the air pollution from traffic in urban areas, so that people can change their behaviors to reduce the air pollution.
\end{abstract}

Keywords-Air pollution; agent-based simulation; traffic

\section{INTRODUCTION}

Nowadays, air pollution is one of the topics of reflection that affects policy-makers around the world. It is a phenomenon that continues to grow overnight because all the human activities contributing to the evolution of new technologies which contribute enormously on this way. In urban areas, like Hanoi City, the main source of pollution is road traffic. Indeed, the means of displacements of the population are among others vehicles that produce particles or pollutants dispersing in the air and strongly denature it chemical composition. Unfortunately, this transformation has a negative effect on the climate and the health of the population. According to [1], air pollution is the alteration of air quality that can be characterized by measurements of chemical, biological or physical gas pollutants, particles, radio logical and sometimes liquid in the air, with consequences detrimental to human health, living beings, climate, or materials.

The main objective of this research work is to propose a decision-making approach on urban air pollution by exploring multi-agent systems under the GAMA platform [2]. This approach will show not only the importance of multi-agent systems, but also allow the population, through awareness sessions, how their daily activities contribute to increasing the pollutants but especially how they could avoid or reduce it considerably. Why the multi-agent systems? Simulation based on multi-agent systems is an approach that is used to reproduce a physical phenomenon from the series of calculations. It leads to the description of the result of this phenomenon, as if it had really taken place because all the actors are represented by agents which embody the capacities of the real actors. This advantage of multi-agents systems will contribute to set up all things understandable. It is an advantage that mathematical models cannot deal with.

Some existing methods are used to model air pollution from road traffic. However, the results produced by these studies are difficult to be interpreted by the general public. The results of these studies are not self-explanatory and require some advanced skills for being interpreted. Among these studies, we noticed the one most related which was set up by Emery et al. [3]. In their study, multi-agents approach has been used to observe the air pollution by taking into account the nature of the automobile park such as gasoline and diesel.

Our approach goes beyond of this study by putting out the principle of the pollutant emission and the mains factors which favor their dispersion by implementing an existing parametric model. The results of this study will be a powerful sensitization tools by the way that everyone will be able to understand and interpret without having a particular advanced skills.

After a detailed study of air pollution modeling methods in urban areas, we chose to build our multi-agent system based on the Operational Street Pollution Model (OSPM) [4]. The OSPM model is one of the standard models used for air pollution modeling in urban areas.

This paper is organized as follows: Section II presents some related works in the field of urban pollution modelling and simulation. Section III presents the proposed model that allows modeling and visualizing the pollution in urban, taking into account traffic factors. Section IV presents the implementation of the model and detail discussion of the simulation results applied to Hanoi city. Finally Section V presents provides some conclusions as well as a discussion about future research.

\section{STATE OF ART}

Several topics were discussed on the modeling of urban air pollution produced by road traffic. Among these subjects, we can note the work of Berkowiz et al. [5] which aimed model air pollution from road traffic at street level using the Operational Street Pollution Model (OSPM). Indeed, the OSPM model, as its name suggests, is a model used in urban areas to model air pollution along streets. In the city of Dijon, Emery et al. [6], [7] have carried out a simulation of 
road traffic based on multi-agent system in order to evaluate automobile air pollution (AAP) from traffic data based on 210 sensors, hence the name of the SCAUP model (MultiAgent Simulation from Urban Counters for Automobile Air Pollution). To realize it, two categories of models have been used, namely the macroscopic model and the microscopic model under the GAMA platform. In the article of Khalesian et al. [8] a micro-simulation approach of multi-agent traffic based on GIS (geographic information system) information was set up to determine atmospheric pollution, in particular the $\mathrm{CO}$ particles generated in a case of traffic congestion. The pollutants taken into account were PM10, SOx and NOx particles. In order to be able to assess and predict the dispersion of air pollution in Delhi city (India), a study was conducted by Kumar et al. [9] using geographic information system (GIS), which is a powerful tool for making more efficient and flexible methods, and a geographic interface simulation system. The study showed that in this city, a large part of the pollution was due to road traffic, either $72 \%$ versus $20 \%$ for industrial sector.

In the sanitary field, pollution scrupulously affects local population health and surrounding areas. Thus, to evaluate this impact in the Baie Area in San Francisco, a team of researchers [10] have implemented a new method that allows to estimating air pollution by modeling the traffic state, traffic induced pollution and dispersion of pollutants along a highway in real time. They used the Gaussian dispersion model, which is a that allows to calculate the concentrations in small spaces. Masoud Fallah Shorshani [11], during his doctoral dissertation developed during his study an evaluation on the feasibility and relevance of a model chain in order to simulate the impact of the traffic on air pollution and runoff, considering traffic account, vehicle emissions, atmospheric transport and processing processes, the watersheds and the processes of leaching and transport by runoff. During his study, he made two models, one static or the boxed model with hourly time steps and the other dynamics or Gaussian plume model, for the traffic and associated pollutants.

In Table I, we highlight the models studied while presenting the strengths and weaknesses of each of these studies.

We note that there are several models and that each of these models is adapted to particular conditions while presenting its advantages and disadvantages. The most criteria discriminant rely on the geographic space to be modeled and also the available data. For our study, from the different analyzes that we carried out, several simulation tools are at our disposal. We have for example MATSim, NETlogo, or GAMA platforms, which offers us great advantages when it comes to integrating data with the Geographic Information System (GIS). As for the pollution dispersion model atmospheric, we will use models that integrate geographical areas information. We have among others the models of the street shaped Canyon, the parallel model CALINE3 developed by Samaranayake et al. [10], the Gaussian plume model already mentioned. The rest of our work will be based on the OSPM model proposed by Berkowiz et al. [5] whose choice was motivated by its characteristics and parameters that cover better our case of study which is the Hanoi city. OSPM model will be presented in the next part.
TABLE I. SUMMARY OF SOME EXISTING WORKS

\begin{tabular}{llll}
\hline Models & & Data & Remarks \\
\hline Operational & Street & -Meteorological & - Usage of weather conditions \\
Pollution & Model & data - Traffic data & - Usage of wind influence \\
(OSPM) [5] & & - Becomes ineffective with a \\
& & & large variation of the wind \\
& & flow \\
& & - Limited to restricted geo- \\
& & graphical areas.
\end{tabular}

- Macroscopic and - Data collected - Consideration of the spatio-
microscopic models from 210 sensors temporal dimension of traffic [6] [7] - Number of vehi- by using sensors

- GAMA Platform cles - Large scale coverage

- Average speed - Requires a lot of data (dis-

placement, localization)

- Not taking into account tem-

poral variations (static)

- High calculation time

- Mandatory data preprocessing

$\begin{array}{ll}\text { Gaussian plum } & \begin{array}{l}\text { Meteorological data } \\ \text { from two years }\end{array} \\ \text { Network (GPM and } & \text { (train \& test) } \\ \text { NN) [12] } & \end{array}$

Consideration of uncontrolled sources

- Consideration of climatic parameters(wind speed, weather, Humidity) - Good prediction: supervised learning with neural networks - Large scale area - Limited number of pollutants taken into account during the study(PM10,SOx,NOx)

\begin{tabular}{lll}
\hline - Model of Gualtieri & - Number of vehi- & - Wind direction and speed \\
and Tartaglia & cles & - solar radiation \\
- Platform : ArcGIS & - Vehicles speed & - Air temperature \\
([8]) & & - Building height \\
& & - Used only for CO (not appli-
\end{tabular}

Used only for CO (not applicable for pollutants involving chemicals process during the dispersion)

\begin{tabular}{|c|c|c|}
\hline $\begin{array}{l}- \\
\text { plume dispersion } \\
\text { pattern(Gaussian } \\
\text { plume dispersion } \\
\text { model)through the } \\
\text { emission factors, } \\
\text { and the traffic } \\
\text { volume traffic [9]; } \\
\text { - Platform: Geomat- } \\
\text { ica10 }\end{array}$ & $\begin{array}{l}\text { Road counting } \\
\text { data } \\
-\quad \text { Meteorological } \\
\text { data } \\
\text { - GIS data }\end{array}$ & $\begin{array}{l}\text { - Flexible } \\
\text { - Large scale application } \\
\text {-Requires a lot of data } \\
\text { - Complex interpretation of } \\
\text { data from satellite observa- } \\
\text { tions }\end{array}$ \\
\hline $\begin{array}{l}\text { - Gaussian disper- } \\
\text { sion model [10] }\end{array}$ & $\begin{array}{l}\text { - Number of vehi- } \\
\text { cles and their aver- } \\
\text { age speed } \\
\text { - Meteorological } \\
\text { data }\end{array}$ & $\begin{array}{l}\text { - Good performance for mea- } \\
\text { surements on intersections } \\
\text { - Real-time modeling } \\
\text { - Integration of the traf- } \\
\text { fic model, weather conditions, } \\
\text { and emission models } \\
\text { - System usable for other } \\
\text { sources of pollution informa- } \\
\text { tion } \\
\text { - Less effective for strong } \\
\text { variations in weather condi- } \\
\text { tions } \\
\text { - Favorable for non-reactive } \\
\text { pollutants }\end{array}$ \\
\hline $\begin{array}{l}\text { - Box model [11] } \\
\text { - Gaussian plume } \\
\text { model }\end{array}$ & $\begin{array}{l}\text { - Traffic data } \\
\text { - Fleet data (gaso- } \\
\text { line, fuel) } \\
\text { - } 26 \text { pollutants } \\
\text { - Using Origin- } \\
\text { Destination Matrix } \\
\text { (O / D) } \\
\text { - Types of vehicles }\end{array}$ & $\begin{array}{l}\text {-Real time vehicles tracking in } \\
\text { the network } \\
\text { - Taking into account numer- } \\
\text { ous pollutants } \\
\text { - High error rate in case of } \\
\text { strong variations of weather } \\
\text { conditions } \\
\text { - Underestimation of some } \\
\text { pollutants like NO2 over short } \\
\text { distances } \\
\text { - No consideration of in- } \\
\text { frastructures in the dispersion } \\
\text { of pollutants (Gaussian plume } \\
\text { model) }\end{array}$ \\
\hline
\end{tabular}




\section{PROPOSED SOLUTION}

\section{A. Overall Model}

Fig. 1 describes a functional model that we designed to describe the air pollution simulation coming from road traffic. In this model, we have essentially three parts. The traffic network which is defined with the vehicles as input data, and the road traffic calculation as output data. In order to calculate the pollutant emission, we take into account the traffic data and the meteorological data in input to observe the pollutants. The last part is the modulation of pollutants dispersion that allows us to observe pollutants dispersion by visualization on traffic area and graphs.

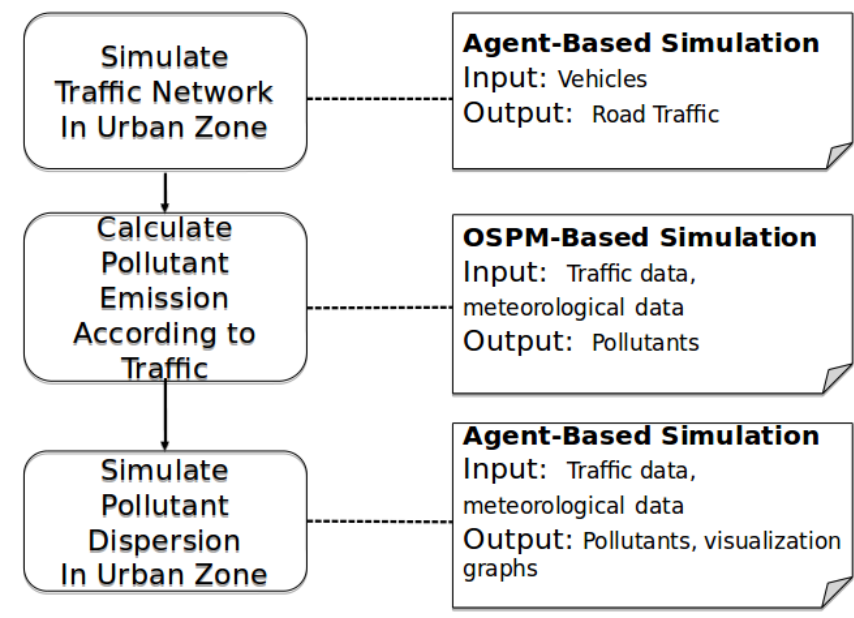

Fig. 1. Functional Model of Proposed Air Pollution Simulation

\section{B. OSPM Model}

1) OSPM model description: The choice of this model is guided by a set of parameters. Each of the existing models has been developed for some specifics conditions.

As a reminder, the main goal of our work is to implement a model that will allow us to simulate air pollution based on multi-agents systems to point out how it grows.

The model to be considered should take into account the geographical dimension to be assessed, the environmental constraints (buildings, trees, etc.), the meteorological constraints (wind, humidity, etc.), the types of pollutants emitted by the traffic and all other phenomena that can influence the dispersion of pollutants in the air. Given the different factors above, and taking into account the existing models, we have chosen to set up a model similar to those used to simulate pollutant levels along urban streets. It's about Operational Street Pollution Model (OSPM) [13].

The Operational Street Pollution Model (OSPM) is a practical model of pollution used in urban areas, particularly at the level of streets in the form of canyon [4]. Developed by the Department of Environmental Sciences of the University of Aarhus in 1989, it has undergone enormous changes over the years [14]. The pollutants concentrations are calculated using a combination of two models [13] that are the plume model and the boxed model.
The plume model calculates the direct contribution of wind to the expansion of pollutants. At this level, with this model, it is assumed that traffic and emissions are uniformly distributed on the entire street. The transmission field is treated as infinitesimal aligned sources line perpendicular to the direction of the wind at the street level. OSPM model does not consider the wind in cross diffusion. Wind direction at street level is supposed to be reflected in relation to the wind at roof level. The box model is used to estimate the contribution of recycled or swirled wind. The implementation of the OSPM model consists of a set of calculations to consider the different conditions and parameters which contribute into the modeling of pollutants dispersion [5].

Referring to the article of Raducan [15], the main assumptions of the model can be summarized as follows:

- All emissions are assumed to be homogeneous along the street.

- When the wind blows perpendicular to the axis of the street, a vortex is form.

- The upwind or upstream side receives a direct contribution from the traffic and some of the pollutants recirculating inside the street.

- The downstream side receives mainly the contribution of recirculating components.

- The concentration of pollutants on both sides of the street is assumed equivalent when the wind blows is zero or blows parallel to the street.

- The direct contribution is calculated using a Gaussian plume model assuming that the pollutants disperse linearly with the distance of the plume.

- The recirculating wind is described using a boxed model.

- The OSPM model can model turbulence in the street assuming it consists of two parts: ambient turbulence (depends on wind speed) and traffic-induced turbulence (which is important when wind speed is weak). In addition, OSPM is a model that integrates a simple photo-chemistry involving nitrogen monoxide (NO), dioxide nitrogen $\left(\mathrm{NO}_{2}\right)$ and $\mathrm{O}_{3}\left(\mathrm{NO}+\mathrm{O}_{3}<=>\mathrm{NO}_{2}\right.$ $\left.+\mathrm{O}_{2}\right)$.

The principle of a pollutant emission by moving vehicles is obtained by summing the particles generated by each type of vehicle while taking into account their emission factor.

$\mathrm{Q}(\mathrm{p})=\sum_{i=1}^{n}\left(\right.$ TypeVehi $*$ FacCoef $\left.f_{p} * P\right)$ where:

- $\quad \mathrm{Q}(\mathrm{p})$ : The total quantity of the pollutant $\mathrm{p}$ generated by all the vehicles during the simulation.

- TypeVehi: The type of vehicle considered.

- FactCoeff (p): The emission factor. Indeed, each vehicle has an emission factor characterizing its contribution level of pollution by type of pollutant.

- P: This is a uniform emission probability that we have defined for each vehicle regardless of the type of pollutant.

So, we can illustrate the model as shown in Fig. 2. 


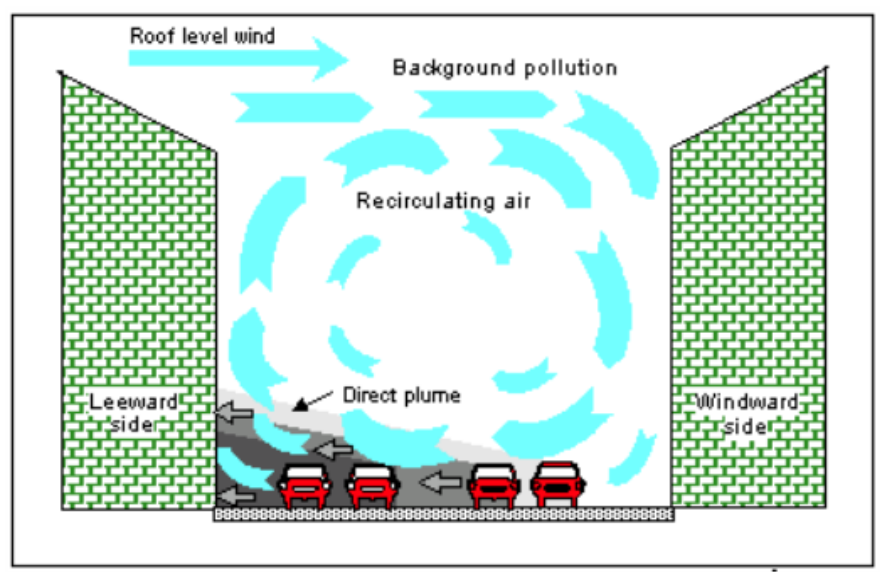

Fig. 2. OSPM figure illustration [4]

2) OSPM model input data: The OSPM model operates primarily based on two categories of input data. These are meteorological data and traffic data.

a) Meteorological data: As shown in the illustrative Fig. 2, meteorological data is strongly involved in the construction of the model. Among these elements, we have the wind which is characterized by its speed and direction, and the temperature of the air. However, this model requires that the wind speed and direction correspond to the flow conditions over the canyon of the street, while the temperature and solar radiation should match the average in all conditions in the canyon of the street.

b) Traffic data: Traffic data is very important for the implementation of the OSPM model. Among these elements, we distinguish vehicles that are categorized by their number, their average speed, and the type of fleet to which it belongs (gasoline or diesel). In addition, we have the heights of the buildings and the width of the concerned street.

\section{MAS (Multi-Agent System) model}

The multi-agent simulation is done through the implementation of a multi-agent system. It is a system composed of several agents performing each activity individually or in interaction with other agents for the purpose of performing or performing an action. This system will simulate behaviors of the real phenomena of everyday life in order to be able to see their impacts or their modes of operation. In our case, the interaction medium is the street on which the vehicles are moving and the desired behavior is how vehicles emit pollutants and how they disperse in the air. As for agents, they represent everything that goes into the constitution of our entire model. Each agent has its own characteristics.

1) Agents extraction: In this part, we define the different agents that enter into the construction of our system. Based on the proposed model (simplified OSPM), we distinguish between two main types of agents: fixed agents and mobile agents.

1) Fixed agents: we distinguish by fixed agent, the set of agents that don't affect movements during the simulation. These agents are, buildings, traffic lights, and trees. Most of these agents are obtained from the corresponding GIS simulation environment.

2) Mobile Agents: So-called mobile agents are the agents that undergo movements during the simulation. According to our system, we can name the wind, the vehicles, and the pollutants that we want to simulate. Thus, we define the characteristics (behaviors and attributes of each agent) as follows:

- Vehicle agent (Fig. 3)

Vehicles are moving agents that move along the street. There are several kinds of vehicles in the city of Hanoi. We distinguish cars, motorbikes, bus, and trucks. Vehicles move with their speed while respecting a certain distance between them. They generate pollutants as they move. However, the type and amount of gas generated depends on the emission factor of the latter. We consider the times at which the inhabitants move less and the hours at which the traffic is denser. Congestion cases will be taken into account during peak hours.

- $\quad$ Pollutant agent (Fig. 3)

Pollutants are the gases generated by vehicles. They are of various natures, for example: $\mathrm{CO}_{2}, \mathrm{NO}_{2}, \mathrm{PM} 2.5$, PM10, etc. More the number of vehicles as the number of pollutants increases, so does the amount of pollutants. In addition to the number of vehicles we also have their average speed. Indeed, when we observe a state of congestion, the average speed of the vehicles decreases considerably thus provoking a high concentration in this area.

- Wind agent (Fig. 3)

Wind is an agent that directly acts on the dispersion of pollutants according to its speed of movement and direction. Higher is its intensity, more rapid is the dispersion.

- $\quad$ Tree agent (Fig. 3)

The trees are placed along the street. They intervene in the limitation or the mitigation of the pollutants dispersion and also in the chemical transformations which certain pollutants undergo $\left(\mathrm{NO}+\mathrm{O}_{3}<=>\mathrm{NO}_{2}+\mathrm{O}_{2}\right.$ ). The reduction of pollution in the canyon streets is much less important than their contributions in its elevation.

The UML diagram 3 below shows the different interaction between agents draw above.

At the end of this study, we present in Fig. 4 the conceptual model that we draw for the multi-agents system simulation.

Our model consists mainly of tree parts. The first outline the input data, the second shows how the OSPM model's designed on GAMA, and the last part sorts the output data.

In the first part, we first build the simplified OSPM model from meteorological data, here only the wind is taken into account, the data of the street, and the data from the road traffic namely the vehicles while taking into account of their category and property as the emission factor. The simplified OSPM 


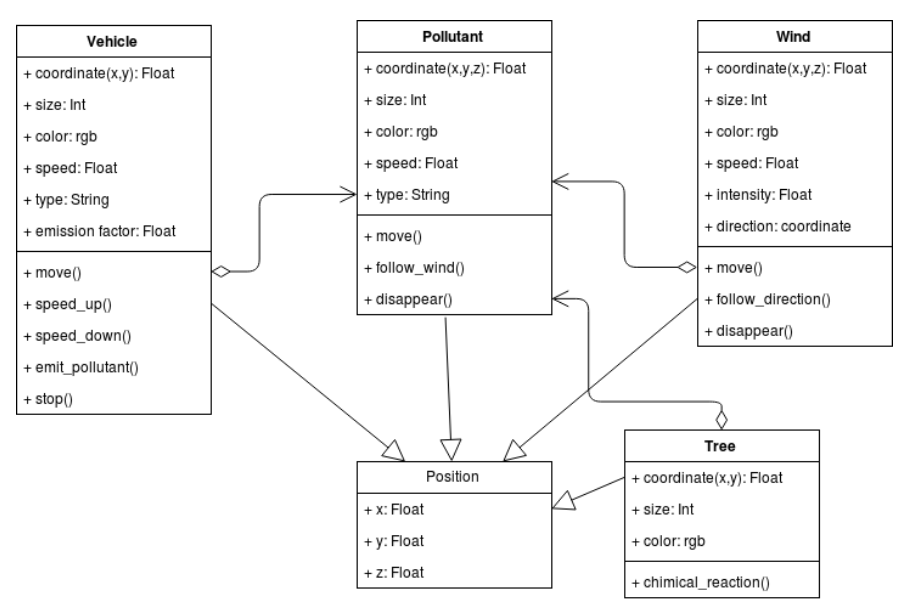

Fig. 3. Agents interaction

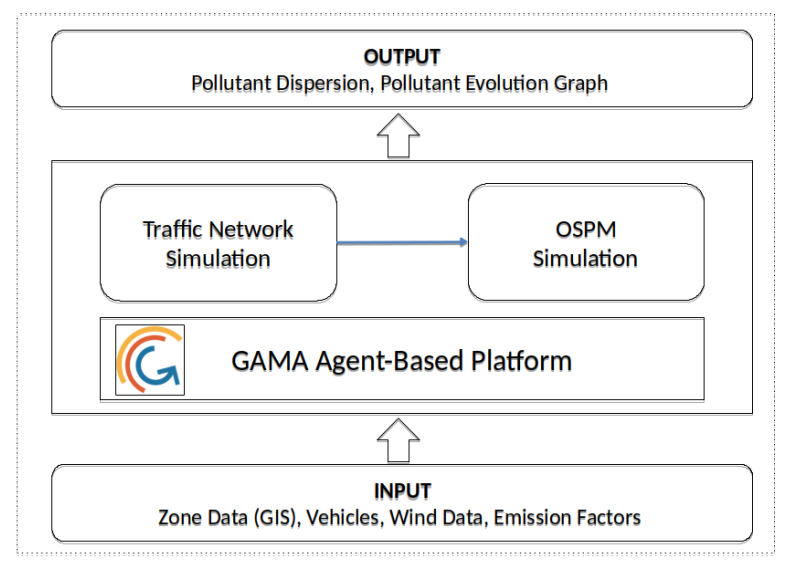

Fig. 4. Design Model of Proposed Air Pollution Simulation

model obtained is implemented under the GAMA platform whose simulation environment is the set of GIS data recovered on OpenStreetMap in the second.

In the last one, we visualize the results produced by the system. The output consists of three types. Firstly it is the evolution and the dispersion of pollutants on the map. Secondly, it is a quantification of the number of particles of each pollutant produced during the simulation, a statistical representation to illustrate the evolution of each pollutant as a function of time and the number of vehicles on the road is presented lastly.

2) Simulation: Simulation is the phase during which we put our system into execution. At the end of it, we get the results produced by our model. These results will allow us to evaluate the performance of the system. Thus, we present the simulation scenarios, the stopping conditions and finally the evaluation parameters.

a) Scenarios of the simulation: The scenarios of the simulation can be summed up in two main parts, namely, the initial phase and the actions repeated during the simulation.

1) Initialization
TABLE II. MODEL EVALUATION CRITERIA

\begin{tabular}{lll}
\hline Input data & Output data \\
\hline The number of vehicles by & $\bullet \quad \begin{array}{l}\text { Quantity of pollutants by } \\
\text { type }\end{array}$ \\
type & $\begin{array}{l}\text { Evolution graphs } \\
\text { The simulation time }\end{array}$ \\
$\begin{array}{l}\text { The speed and direction of } \\
\text { the wind }\end{array}$ & $\bullet \quad \begin{array}{l}\text { Illustration of polluted areas } \\
\text { - }\end{array}$
\end{tabular}

- Import the map (GIS contained in the shape file);

- Initialize the number of vehicles to 0

- Generate the wind;

- Create vehicles with different destinations;

- Assign an energy to each vehicle;

- Create the trees.

2) Repeat

- Vehicles travel on different roads;

- Each vehicle emits pollutants

- The energy of each vehicle decreases when its moving. When its energy ends, its traffic stops;

- The traffic lights change color;

- The wind moves in one direction to regularize the traffic;

- Pollutants move in the direction of the wind.

b) Simulation stop conditions: Stopping the simulation is conditioned by the observed results

c) Evaluation parameters: Rating parameters are the parameters on which we will base ourselves to know if the model is good or not. They are located on both sides namely at the input of the model and at the output of the model. When we submit input data to the model, results are expected at the output. However, the results can corroborate with those expected or not. So we define our parameters as shown in the Table II.

\section{IMPLEMENTATION AND EXPERIMENTAL ON ROAD TRAFFIC SIMULATION}

\section{A. Input Data Description}

As with any parametric model, we still need input data for validation. The data used are:

- The vehicles: In our model, we mainly design four types of vehicles that are: trucks, personal cars, bus and motorbikes. The choice of these data is guided by the reality of Hanoi. Indeed, the city of Hanoi is mainly driven by these four types of vehicles. Each type of vehicle is affected by an emission factor ([16], p.193) which able to determine the participation of each one in the emission of the pollutants. We considered four types of pollutants which are the most caused by road traffic. Table III presents the contribution of each of the vehicles cited in the emission of pollutants.

- The wind: The wind is one of the main elements that enters into the dispersion of pollutants. When we applied wind, pollutants are systematically redirected towards the direction of the latter and are dispersed 
TABLE III. EMISSION FACTOR

\begin{tabular}{ccc}
\hline Type of vehicle & Emission Factor(\%) & Pollutant \\
\hline Truck & 0.80 & $\mathrm{PM}$ \\
\cline { 2 - 3 } & 2.75 & $\mathrm{CO}$ \\
\cline { 2 - 3 } & 11.00 & $\mathrm{NO}_{x}$ \\
\cline { 2 - 3 } & 0.40 & $\mathrm{SO}_{2}$ \\
\cline { 2 - 3 } & 1.50 & $\mathrm{PM}$ \\
\cline { 2 - 3 } & 3.10 & $\mathrm{CO}$ \\
\cline { 2 - 3 } & 7.60 & $\mathrm{NO}_{x}$ \\
\hline Car & 0.64 & $\mathrm{SO}_{2}$ \\
\cline { 2 - 3 } & 0.10 & $\mathrm{PM}$ \\
\cline { 2 - 3 } & 3.62 & $\mathrm{CO}$ \\
\hline Motorbike & 1.50 & $\mathrm{NO}_{x}$ \\
\cline { 2 - 3 } & 0.17 & $\mathrm{SO}_{2}$ \\
\cline { 2 - 3 } & 0.10 & $\mathrm{PM}$ \\
\cline { 2 - 3 } & 3.62 & $\mathrm{NO}_{x}$ \\
\cline { 2 - 3 } & 0.30 & $\mathrm{SO}_{2}$ \\
\cline { 2 - 3 } & 0.03 & \\
\hline
\end{tabular}

along environment. Thus, even places where there is no consequent traffic, are polluted due to transport by the wind. The main features that distinguish are its intensity and direction.

- The trees: Like the wind, the trees intervene in the dispersion of the pollutants firstly and also favor the generation of certain pollutants like the $\mathrm{CO}_{2}$ by the principle of the photosynthesis.

- The map: It is our simulation environment. We extracted part of the city of Hanoi from OpenStreetMap that we imported into QGIS to get the shape file, the shape file we used under the GAMA platform. Fig. 5 saw us the selection on OpenStreetMap.

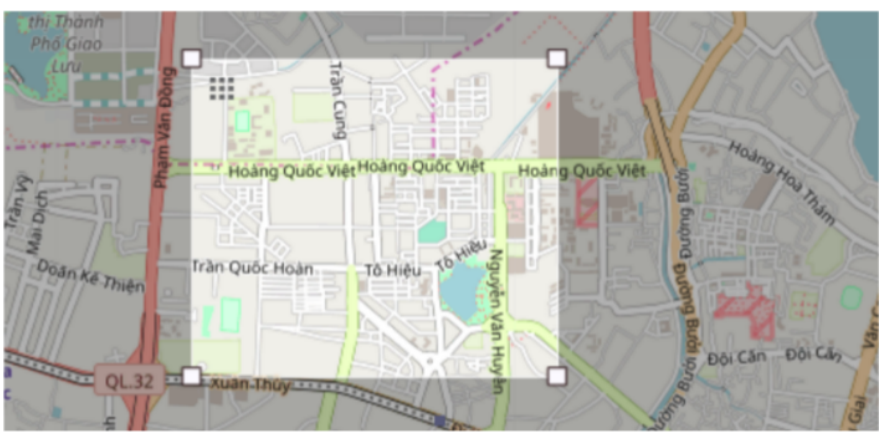

Fig. 5. Simulation environment

In addition to these data, taking into account the complete model proposed in the second part, there are other parameters that may be use but that we haven't used in the simplified model. It is:

- the size of the buildings that are located along each street;

- the width of the street: it allows to estimate the number of vehicles that it can contain and to deduce also the cases of congestion.

\section{B. Discussion}

Initially, i.e. before starting the simulation, our environment is as shown in Fig. 6. In order, we show in Fig. 7, the different vehicles on the road during the traffic.

Fig. 8 presents all the agents present in our environment once the simulation is launched. As mentioned above, we have

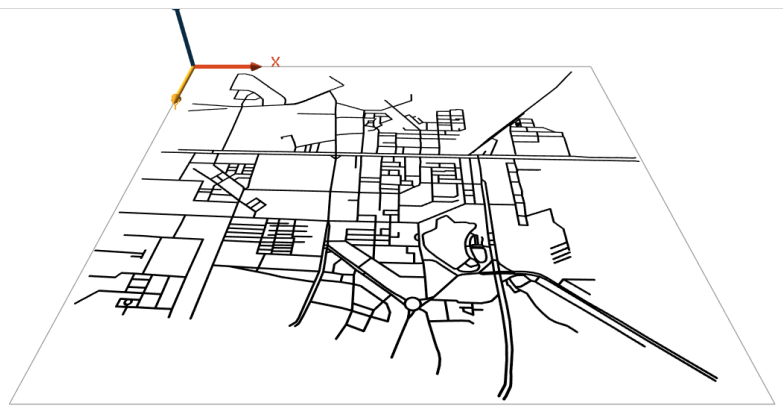

Fig. 6. Initial environment

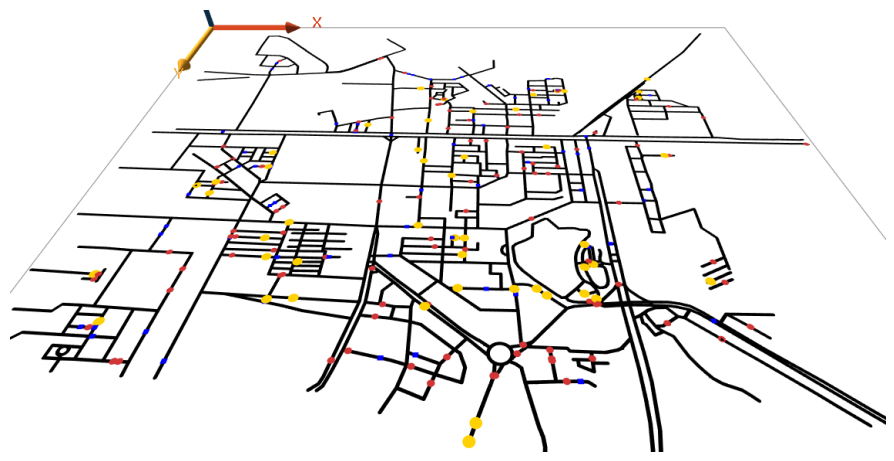

Fig. 7. Traffic network

considered four types of vehicles that are personal cars, bus, trucks and motorcycles. As for pollutants, we also note the four types of pollutants that are $\mathrm{NO}_{x}, \mathrm{CO}, \mathrm{PM}$ and $\mathrm{SO}_{2}$ that we have chosen to take into account in our system. As indicated

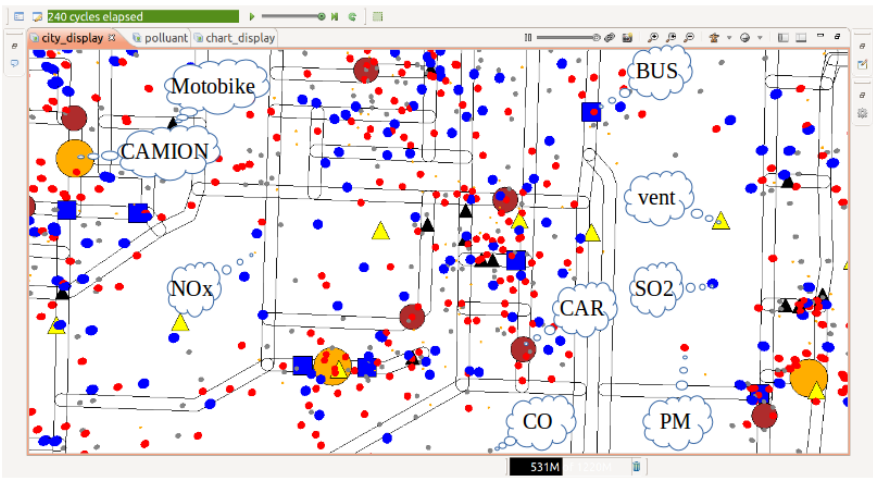

Fig. 8. Agent list

in scenarios section, we simulate the dispersion of pollutants mainly taking into account three cases.

1) The first case is one in which we consume a fairly stable wind. These are the periods during which the wind direction remains unstable.

We find that pollutants are dispersed around the environment, but without forming an agglutination at a given place, for example. In addition, areas where there are no vehicles are also affected because pollutants are dispersed little everywhere: this is explained by the instability of the wind direction. 
2) The second case is when we consider that sometime, the wind has a direction and moves with a speed that can also be varied. We took two different directions in this parity. The results obtained are shown in Fig. 9 where we present how pollutants evolve in the direction of the wind.

We notice that when the wind moves with a precise direction, one of the very important points is that even the places where there is no heavy traffic for example the residential areas are flooded with large quantities of pollutants. The wind therefore favors not only the dispersion, but also pollution transport from places with heavy traffic to places almost 'safely'.

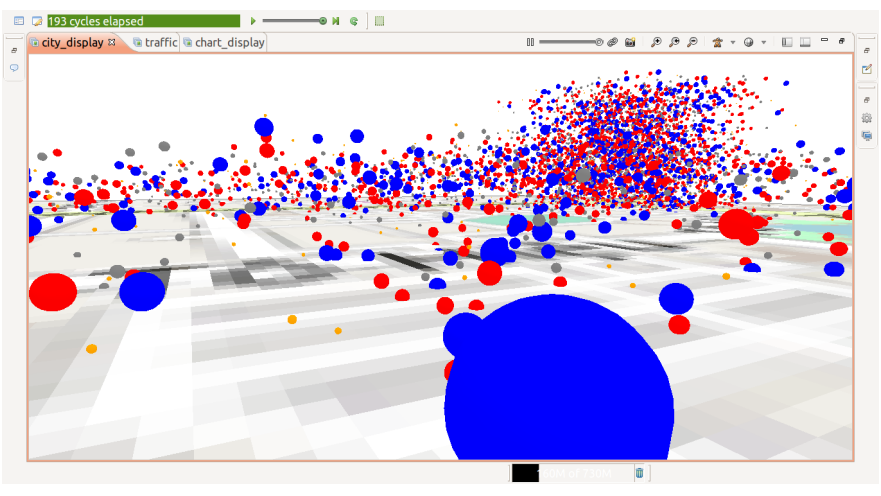

Fig. 9. Pollution map when applying a directional wind

3) The third case we have illustrated is traffic congestion. Indeed, congestion related to road traffic is one of the recurrent phenomena that we see everyday, especially in peak hours. Several situations can explain congestion. It may be due either to the hours of descents or days start times during which the number of vehicle becomes very important on the road.

We have seen with the results obtained an increase in the level of pollutants at intersections. Indeed, the number of vehicles is considerable in these places and the flow of traffic becomes very slow. Each vehicle moves at a very low speed and releases a lot of particles causing a lot of pollution in these places. We present a simulation case in Fig. 10.

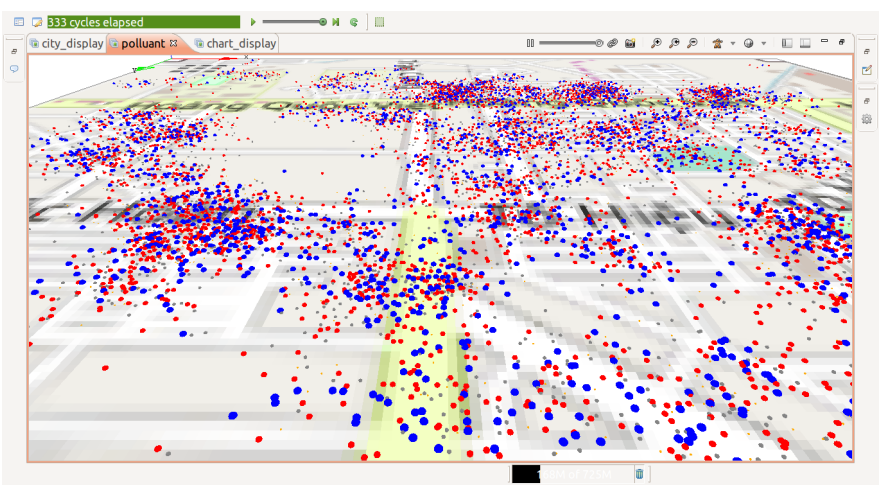

Fig. 10. Pollution map in a case of congestion.

To better track the impact of traffic on the level of pollution, we proceeded to establish a followup in statistical form. During the simulation phase,
TABLE IV. EXPERIMENTAL RESULTS

\begin{tabular}{cccccc} 
& & \multicolumn{4}{c}{ Quantity of pollutant (count) } \\
\cline { 3 - 6 } Freq(vehi/s) & Nb_Vehi & $\mathrm{PM}$ & $\mathrm{CO}$ & $\mathrm{NO}_{x}$ & $\mathrm{SO}_{2}$ \\
\hline 5 & $\tilde{3} 60$ & 1413 & 2353 & 3254 & 1807 \\
\hline 10 & 720 & 2657 & 4843 & 6512 & 3642 \\
\hline 15 & 1008 & 3859 & 7104 & 9611 & 5313 \\
\hline
\end{tabular}

we progressively assessed the number of particles released for all vehicles indiscriminately over time. We illustrated our experiment in two cases. Firstly, we considered the situation where the traffic is high, during the open days in the week. The second case was highlighted in the case of the week-end. Indeed, the traffic during the week-end is more important than the others days of the week.

Fig. 11 presents the evolution of each type of pollutant during the simulation.

Table IV sums the different cases of simulations that we made. This process allows us to even quantitatively the evolution of pollutants and their dispersion as a function of time and the number of vehicles in motion.

To better situate our experimentation, we played mainly on two characteristics parameters: the speed of creation of the vehicles, and the simulation time. For this, we set a uniform simulation time of 250 cycles while playing on the frequency of the vehicles generation. The creation frequencies of the vehicles used are 5, 10 and 15 vehicles/s.

The quantity of pollutants increases over time with the number of vehicles. Moreover, while maintaining respectively the same simulation time, in the three cases of different frequencies, the increase in the pollution level goes hand in hand with the latter. This clearly explains that the number of vehicles in circulation induces directly on the quantity of pollutants produced. Moreover, with generating the vehicles in a fixed time, that shows us how the quantity of pollutants stay stable at the end of this time. During the experimentation, we set the time at 500 seconds, 334 vehicles has been generated and were running at this time. The stability of the pollution at $\mathrm{t}=700 \mathrm{~s}$ is due by the fact that all vehicle are stopped running because of the lack of energy. That allows to show the impact of traffic in the increase of pollution.

In this part we can notice from the results obtained that the wind is a very important factor that enters into the principle of the dispersion of pollutants. When it moves with a precise direction, it leads to a transportation of pollutants in the residential areas as well as in places not involving high traffic. In addition to that, we were able to show that more the number of vehicles is high in a place, the traffic become slower and contribute to increase the level of pollution.

As a reminder, the main objective of our work is the establishment of a decision support system on urban air pollution through the exploration of multi-agent systems. The results obtained from this study can be used in four (04) major points such are:

- A practical demonstration tool for a public awareness. 


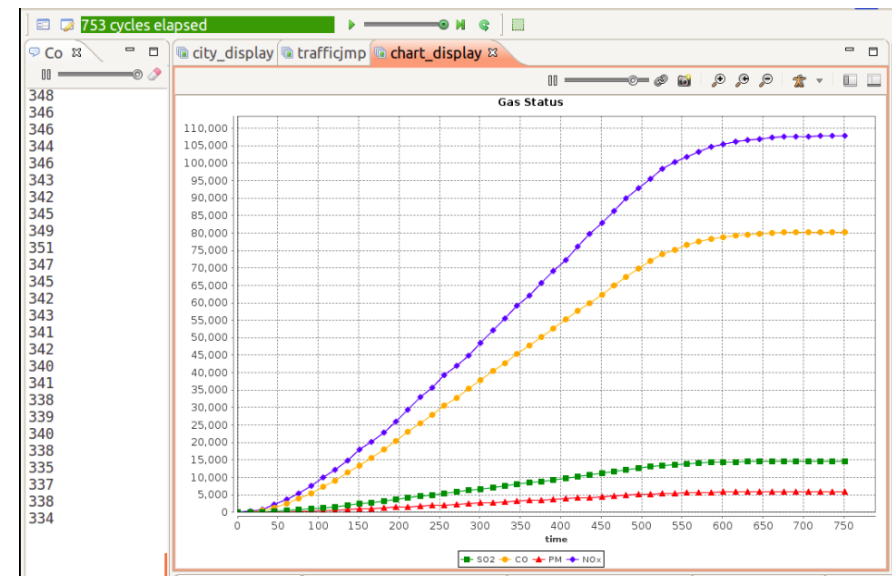

Fig. 11. Evolution of pollutants during the time

During an awareness session, it is more effective to present the public with simulation cases close to the reality. Indeed, the majority of the population are not able to interpret mathematical results, hence the ineffectiveness of modeling methods based on complex results. People need more or less real illustrations to understand the impact or the spread of their practices on air pollution.

- Better control over the parameters favoring the dispersion of pollutants. In our study, we were able to highlight the impact of the wind on the dispersion mainly in two cases namely in the presence of a stable wind and wind which have a high speed with a given direction. This point is very important because it allows to have an idea in advance of the risk areas during the different seasons of the year thus allowing to take adequate preventive measures for the concerned population protection.

- Explicitly exposing the most polluted places. During our study, we highlighted the most exposed areas to air pollution. This is the case of traffic congestion. As indicated above, in case of congestion, the flow of traffic increases, thus inducing a strong release of pollutants at these locations. In the case of non-dispersion or slow dispersion, the concern environments become more and more unbearable due to the pollutants in these places. This is the perfect case when we are expecting a stable wind without direction.

- An highlight of the impact of the car fleet on air pollution. As stated by Justin Emery [6], the nature of the car fleet has an important impact on air pollution. An awareness of the types of vehicles based on their fleet will allow population to have what kind of vehicle is needed to help fight against air pollution.

\section{CONCLUSION}

During this work, we are able to study the principles of emission and dispersion of pollutants released by vehicles in urban area (the case of Hanoi City). In the section devoted to the theoretical studies, we have evaluated different techniques and models already existing for urban air pollution modeling.
After this exploration, we proposed to simulate with the OSPM model which is one of the most explicit pollution model for urban case studies.

Among the emission factor's (Table III), the number of vehicles is one the important factors which affects directly the quantity of pollutants. About the principle of dispersion, our work are focused on the impact of the wind, to show how the wind intervenes or contributes on the pollutants dispersion. We designed three mains cases where we saw the results when applying a wind with a specific direction (Fig. 9), and a stable wind by taking into account a case of traffic jam (Fig. 10). The results obtained allow us to highlight the impact of road traffic on air pollution and also phenomena that favor their dispersion. These illustrations will undoubtedly show the target audience in an understandable way the impact of the different vehicles on the air pollution as well as the contribution of the wind in its evolution.

However, much remains to be done because the model we have achieved during the simulation is only a simplified version of the original OSPM model.

As a perspective, for an improvement of the work carried out, we recommend the application or the implementation of the following points:

- A representation of pollutants in the form of cloud. Indeed, in the work already done, the pollutants that we simulated, each has been represented as an agent, which weighs down the system. In order to make it optimal, it would therefore be necessary to represent the whole in the form of a cloud while varying the color of the latter according to the amount of each of the pollutants in order to be able to highlight them. This approach will make it possible to summarize all the pollutants in a single agent, which will contribute to greatly reduce the calculation time.

For its implementation, one of the possible options would be to cover the entire area with a grid. Then, you will have to map between each cell of the grid with the vehicles that are located. As each vehicle produces several categories of pollutants, we can color each cell according to the types of vehicles that are there from the color assigned to each of the pollutants. From there, we could have a multi-colors grid whose color varies with the passage of time according to the flow that knows the traffic and even to locate the most polluted places.

- Increase the number of vehicles during the simulation. When the number of vehicles is important, the results will be even more convincing and can help us draw more reliable conclusions.

- Consider the car park. It is undoubtedly the type of fuel (petrol, diesel) that the vehicle uses greatly induces the types and amount of pollutants released by the vehicle. It would therefore be necessary to distinguish them in order to obtain finer and more plausible results.

- Provide field data for model feeding and validation. Indeed, the results that we obtain at the output of our model are related to the data used for the simulation 
of the model. Field data will be an additional factor a model close to reality. For example, vehicle types count, measurements on meteorological data (wind, humidity, etc.), and all the other parameters of the original model (OSPM). As for the validation of the model, measurements of funds taken in the field will make it possible to make comparisons with the results obtained at the exit of the model.

\section{REFERENCES}

[1] D. Duong/CVN, "Hanoi : vers la modernisation du parc de véhicules en 2018 - le courrier du VietNam."

[2] A. Grignard, P. Taillandier, B. Gaudou, D. A. Vo, N. Q. Huynh, and A. Drogoul, "Gama 1.6: Advancing the art of complex agent-based modeling and simulation," in PRIMA 2013: Principles and Practice of Multi-Agent Systems (G. Boella, E. Elkind, B. T. R. Savarimuthu, F. Dignum, and M. K. Purvis, eds.), (Berlin, Heidelberg), pp. 117-131, Springer Berlin Heidelberg, 2013.

[3] J. Emery, N. Marilleau, N. Martiny, T. Thévenin, T. Nguyen-Huu, M. A. Badram, A. Grignard, H. Hbdid, A.-M. Laatabi, and S. Toubhi, "Marrakair: une simulation participative pour observer les émissions atmosphériques du trafic routier en milieu urbain," in Treizièmes Rencontres de Théo Quant, (Besançon, France), May 2017.

[4] O. Hertel, Operational Street Pollution Model (OSPM). Evaluation of the model on data from St. Olavs street in Oslo. Danmarks Miljøundersøgelser, 1989.

[5] R. Berkowicz, O. Hertel, S. Larsen, N. Sørensen, and M. Nielsen, "Modelling traffic pollution in streets," National Environmental Research Institute, Roskilde, Denmark, vol. 10129, no. 10136, p. 20, 1997.

[6] J. Emery, La ville sous électrodes: de la mesure à l'évaluation de la pollution atmosphérique automobile.: vers une simulation multi-agents du trafic routier en milieu urbain. PhD thesis, Dijon, 2016.
[7] J. Emery, N. Marilleau, N. Martiny, T. Thévenin, and J. Villery, "L'apport de la simulation multi-agent du trafic routier pour l'estimation des pollutions atmosphériques automobiles," in Douzièmes Rencontres de Théo Quant, 2015.

[8] M. Khalesian, P. Pahlavani, and M. Delavar, "Gis-based multi-agent traffic micro simulation for modelling the local air pollution," 2008.

[9] A. Kumar, R. K. Mishra, and S. Singh, "Gis application in urban traffic air pollution exposure study: a research review," Suan Sunandha J Sci Technol, vol. 2, no. 1, pp. 1-13, 2014.

[10] S. Samaranayake, S. Glaser, D. Holstius, J. Monteil, K. Tracton, E. Seto, and A. Bayen, "Real-time estimation of pollution emissions and dispersion from highway traffic," Computer-Aided Civil and Infrastructure Engineering, vol. 29, no. 7, pp. 546-558, 2014.

[11] M. Fallah Shorshani, Modélisation de l'impact du trafic routier sur la pollution de l'air et des eaux de ruissellement. $\mathrm{PhD}$ thesis, Paris Est, 2014.

[12] S. Ghazi, J. Dugdale, and T. Khadir, "Modelling air pollution crises using multi-agent simulation," in System Sciences (HICSS), 2016 49th Hawaii International Conference on, pp. 172-177, IEEE, 2016.

[13] P. Kastner-Klein, R. Berkowicz, and E. Plate, "Modelling of vehicleinduced turbulence in air pollution studies for streets," International Journal of Environment and Pollution, vol. 14, no. 1-6, pp. 496-507, 2000.

[14] K. E. Kakosimos, O. Hertel, M. Ketzel, and R. Berkowicz, "Operational street pollution model (ospm)-a review of performed application and validation studies, and future prospects," Environmental Chemistry, vol. 7, no. 6, pp. 485-503, 2010.

[15] G. Raducan, "Pollutant dispersion modelling with ospm in a street canyon from bucharest," Romanian Report in Physics, vol. 60, pp. 1099-1114, 2008.

[16] N. T. Hung, Urban air quality modelling and management in Hanoi, Vietnam. National Environmental Research Institute, Aarhus University, 2010. 